\title{
A distinct profile of tryptophan metabolism along the kynurenine pathway downstream of toll-like receptor activation in irritable bowel syndrome
}

\author{
Gerard Clarke $1,2,3 *$, Declan P. McKernan ${ }^{4}$, Gabor Gaszner ${ }^{5}$, Eamonn M. Quigley ${ }^{1,6}$, John F. Cryan ${ }^{1,7}$ and \\ Timothy G. Dinan ${ }^{1,5}$
}

${ }^{1}$ Alimentary Pharmabiotic Centre, University College Cork, Cork, Ireland

2 Department of Pharmacology and Therapeutics, University College Cork, Cork, Ireland

${ }^{3}$ School of Pharmacy, University College Cork, Cork, Ireland

${ }^{4}$ Department of Pharmacology and Therapeutics, University College Galway, Galway, Ireland

${ }^{5}$ Department of Psychiatry, University College Cork, Cork, Ireland

${ }^{6}$ Department of Medicine, University College Cork, Cork, Ireland

7 Department of Anatomy and Neuroscience, University College Cork, Cork, Ireland

\section{Edited by:}

Angelo A. Izzo, University of Naples

Federico II, Italy

Reviewed by:

Thomas J. Connor, Trinity College, Ireland

Martin Diener, University Giessen, Germany

*Correspondence:

Gerard Clarke, Alimentary

Pharmabiotic Centre, University

College Cork, Cavanagh Pharmacy

Building, Office UG04, Cork, Ireland.

e-mail: g.clarke@ucc.ie
Irritable bowel syndrome (IBS), a disorder of the brain-gut axis, is characterised by the absence of reliable biological markers. Tryptophan is an essential amino acid that serves as a precursor to serotonin but which can alternatively be metabolised along the kynurenine pathway leading to the production of other neuroactive agents. We previously reported an increased degradation of tryptophan along this immunoresponsive pathway in IBS. Recently, altered cytokine production following activation of specific members of the toll-like receptor (TLR) family (TLR1-9) has also been demonstrated in IBS. However, the relationship between TLR activation and kynurenine pathway activity in IBS is unknown. In this study, we investigated whether activation of specific TLRs elicits exaggerated kynurenine production in IBS patients compared to controls. Whole blood from IBS patients and healthy controls was cultured with a panel of nine different TLR agonists for $24 \mathrm{~h}$. Cell culture supernatants were then analyzed for both tryptophan and kynurenine concentrations, as were plasma samples from both cohorts. IBS subjects had an elevated plasma kynurenine:tryptophan ratio compared to healthy controls. Furthermore, we demonstrated a differential downstream profile of kynurenine production subsequent to TLR activation in IBS patients compared to healthy controls. This profile included alterations at TLR1/2, TLR2, TLR3, TLR5, TLR7, and TLR8. Our data expands on our previous understanding of altered tryptophan metabolism in IBS and suggests that measurement of tryptophan metabolites downstream of TLR activation may ultimately find utility as components of a biomarker panel to aid gastroenterologists in the diagnosis of IBS. Furthermore, these studies implicate the modulation of TLRs as means through which aberrant tryptophan metabolism along the kynurenine pathway can be controlled, a novel potential therapeutic strategy in this and other disorders.

Keywords: irritable bowel syndrome, kynurenine pathway, toll-like receptors, tryptophan, IDO, cytokine

\section{INTRODUCTION}

The diagnosis of irritable bowel syndrome (IBS), a highly prevalent functional gastrointestinal disorder (FGID), is currently made based on the presence of a characteristic symptom profile (abdominal pain/discomfort, bloating/distension, alterations in defecatory function) in the absence of a demonstrable organic disease of the gastrointestinal tract (GIT; Drossman and Dumitrascu, 2006). This diagnostic scheme reflects the lack of reproducible biological markers of this heterogeneous disorder, a serious impediment to advancing our understanding of its pathophysiology (Clarke et al., 2009b). The concept of IBS as a disorder of the brain-gut axis is now generally accepted and this has facilitated some progress in the area (Ohman and Simren, 2007, 2010). Recently, indices of a low grade immune activation have been reported in IBS, including elevations in circulating cytokines (Dinan et al., 2006; Liebregts et al., 2007; Dinan et al., 2008; Scully et al., 2010) and pro-inflammatory polyunsaturated fatty acids (Clarke et al., 2010) as well as evidence of enhanced immune cell activation, both systemically (Ohman et al., 2009a,b) and locally within the GIT (Cremon et al., 2009).

A growing appreciation of the potential impact of this increased inflammatory state on the brain-gut axis (Quigley, 2006; Dantzer et al., 2008; O'Malley et al., 2011) as well as an improved understanding of the influence of the GIT on mood and cognition (Forsythe et al., 2010; Grenham et al., 2011; Mayer, 2011; Bercik et al., 2012; Kennedy et al., 2012), has led to an exploration of a potential role for tryptophan and its associated metabolic 
pathways in IBS. Tryptophan is an essential amino acid that serves as a precursor to serotonin (5-HT), a key neurotransmitter within both the enteric nervous system (ENS) and central nervous system (CNS; Ruddick et al., 2006; Forsythe et al., 2010). An alternative and physiologically dominant fate for tryptophan is degradation along the kynurenine pathway leading to the production of neuroprotective compounds like kynurenic acid and neurotoxic compounds like quinolinic acid (Schwarcz and Pellicciari, 2002). Dysregulation of tryptophan metabolism is thus poised to impact on mood and cognition within the CNS as well as secretion, motility, and perception in the ENS (Crowell, 2004; Forsythe et al., 2010). Crucially, indoleamine-2.3-dioxygenase (IDO), one of two key primary kynurenine pathway enzymes, is immunoresponsive and studies, to date, have indicated an increase in IDO activity in both male and female subjects with IBS (Fitzgerald et al., 2008; Clarke et al., 2009a).

Mechanistic insights into the inflammation observed in IBS and the associated downstream consequences are currently lacking. Toll-like receptors (TLRs) are pattern recognition receptors integral to the functioning of the innate immune system and respond to a variety of bacterial and viral cell components, resulting in increased production of inflammatory cytokines (Takeuchi and Akira, 2010). Recent evidence from both relevant animal models and biopsies from IBS sufferers have demonstrated altered expression of certain TLRs in the colonic mucosa (McKernan et al., 2009; Brint et al., 2011). Increased TLR2 expression on blood monocytes in IBS patients has also been reported (Ohman et al., 2012). Moreover it has recently been demonstrated that IBS patients have a distinct pattern of peripheral TLR activity as indicated by measurements of cytokine production following whole blood stimulation (McKernan et al., 2011). Although it is known that TLR activation can lead to alterations in IDO expression (Mahanonda et al., 2007), the potential consequences for tryptophan metabolism in IBS remain unknown.

In this study we investigated the potential functional consequences of TLR activation on brain-gut axis signaling in terms of perturbations in tryptophan metabolism. This was based on the hypothesis that IBS patients would exhibit a downstream pattern of tryptophan degradation along the kynurenine pathway subsequent to activation of TLRs with their specific ligands that was distinct from healthy control subjects.

\section{MATERIALS AND METHODS STUDY POPULATION}

The study protocol was approved by the University College Cork (UCC) Clinical Research Ethics Committee. IBS patients were recruited from a university IBS database comprised of individuals who had either attended gastroenterology clinics at Cork University Hospital (CUH) or had responded to direct advertisement on the university campus or a local newspaper regarding participation in IBS research. Thirty seven healthy controls were recruited from staff at both UCC and CUH. Twenty five individuals aged between 18 and 65 years who satisfied Rome II criteria for IBS and in whom organic gastrointestinal diseases and clinically significant systemic diseases had been excluded, were considered for inclusion in the study. Subjects who had undergone any abdominal surgery, with the exception of hernia repair and appendectomy, were also excluded. No postinfectious IBS (PI-IBS) subjects were included in the recruitment and IBS patients were not selected on the basis of predominant bowel habit, although this was recorded according to the Rome II classifications. All subjects completed a questionnaire to assess both IBS severity (Francis et al., 1997) and current mood (Spitzer et al., 1999).

\section{BIOLOGICAL ASSAYS}

Fifteen milliliters of whole blood was collected between 11:00 and 13:00 hours from each healthy control and IBS patient. Collected whole blood $(15 \mathrm{~mL})$ was added to an equal volume of Histopaque 1077 (Sigma, St Louis, MO, USA) in a sterile $50 \mathrm{~mL}$ tube and centrifuged at $400 \times g$ for $30 \mathrm{~min}$ at room temperature. Plasma on the upper layer was transferred to a separate tube and stored at $-80^{\circ} \mathrm{C}$ for future analysis. Collected whole blood $(2 \mathrm{~mL})$ was diluted 1:10 in Dulbecco's Modified Eagle's Medium (DMEM; Gibco, Dublin, Ireland). Blood was aliquoted into 24 well plates and cultured in a $37^{\circ} \mathrm{C}$ incubator with $5 \% \mathrm{CO}_{2}$. Each blood sample was cultured in duplicate in DMEM cell culture medium supplemented with $10 \%$ Fetal Calf Serum (Sigma, Dublin, Ireland) with or without the following TLR ligands from a Human TLR agonist kit (Invivogen, San Diego, CA, USA) for $24 \mathrm{~h}$ :

TLR1/2-Palmitoyl-3-cysteine-serine-lysine 4 (Pam3Cys); TLR2heat-killed Listeria monocytogenes (HKLM); TLR3-Polyriboinosinic polyribocytidylic acid (Poly I:C); TLR4-Lipopolysaccharide (LPS); TLR5-Salmonella typhimurium Flagellin; TLR6/2-FSL-1; TLR7Imiquimod; TLR8-ssRNA40; TLR9-ODN2006. Agonists were reconstituted in endotoxin free water (supplied in kit) to a final concentration of $1 \mu \mathrm{g} \mathrm{mL}^{-1}$ except for HKLM ( $10^{8}$ cells) and Poly I:C $\left(10 \mu \mathrm{g} \mathrm{mL}^{-1}\right)$. Subsequently, supernatants from both untreated and stimulated cells were aspirated and stored at $-80^{\circ} \mathrm{C}$ for future analysis.

\section{HPLC ASSAY FOR TRYPTOPHAN AND KYNURENINE}

Tryptophan and kynurenine were determined by high performance liquid chromatography (HPLC): this involved using a system comprising a Waters 510 pump (Waters Ireland, Dublin, Ireland), 717plus cooled autosampler, a 996 PDA detector, a Hewlett Packard 1046A Fluorescent Detector (Waters Ireland, Dublin, Ireland), a waters bus SAT/IN module and a croco-cil column oven. System components were used in conjunction with Waters Empower software (Waters Ireland, Dublin, Ireland). All samples were injected onto a reversed phase Luna $3 \mu \mathrm{C} 18(2) 150 \times 2 \mathrm{~mm}$ column (Phenomenex, Macclesfield, UK), which was protected by Krudkatcher disposable precolumn filters and security guard cartridges (Phenomenex). HPLC grade acetonitrile, acetic acid, and perchloric acid were obtained from Fisher Scientific Ireland (Dublin, Ireland). The analysis method was based on that by Herve et al. (1996). The mobile phase consisted of $50 \mathrm{mmol} \mathrm{L}^{-1}$ acetic acid, $100 \mathrm{mmol} \mathrm{L}^{-1}$ Zinc Acetate with 3\% (v/v) acetonitrile and was filtered through a $0.45 \mu \mathrm{m}$ Millipore filter (AGB, Dublin, Ireland) and vacuum degassed prior to use. Separations were achieved by isocratic elution at $0.3 \mathrm{~mL} \mathrm{~min}^{-1}$. The fluorescent detector was set to an excitation wavelength of $254 \mathrm{~nm}$ and an emission wavelength of $404 \mathrm{~nm}$. The PDA detector start wavelength was $210 \mathrm{~nm}$ and the end wavelength was $400 \mathrm{~nm}$ with chromatogram extraction at $330 \mathrm{~nm}$. Working standard dilutions 
were prepared from millimolar stock solutions of each standard and stored at $-80^{\circ} \mathrm{C}$ until required for analysis. Samples were deproteinized by the addition of $20 \mu \mathrm{L}$ of $4 \mathrm{~mol} \mathrm{~L}^{-1}$ perchloric acid to $200 \mu \mathrm{L}$ of plasma spiked with 3-nitro-L-tyrosine as internal standard. Twenty microliters of either sample or standard was injected onto the HPLC system and chromatograms generated were processed using Waters Empower software. Analytes were identified based on their characteristic retention time and their concentrations determined using Analyte:Internal standard peak height ratios; these were measured and compared with standard injections which were run at regular intervals during the sample analysis. Results were expressed at $\mathrm{ng}$ analyte per $\mathrm{mL}$ of supernatant/plasma.

\section{STATISTICS}

The sample size was determined by a power calculation based on our previous data and aimed at detecting differences between IBS patients and controls at the 0.05 level. Data was expressed as mean \pm SEM. Statistical analysis was carried out using SPSS 18 for Windows (SPSS, Inc., Chicago, IL, US). Plasma tryptophan, kynurenine, and the kynurenine:tryptophan ratio were compared using an unpaired two-tailed Student's $t$-test and differences considered significant at the $p<0.05$ level. Bonferroni corrections for multiple comparisons were applied as required. TLR agonist induced alterations in, kynurenine and the kynurenine:tryptophan ratio were determined using a two-way ANOVA and by Bonferroni post hoc tests.

\section{RESULTS}

\section{BASELINE CHARACTERISTICS}

There were no significant differences between IBS patients $(5 \mathrm{M}$, $20 \mathrm{~F})$ and controls $(13 \mathrm{M}, 25 \mathrm{~F})$ in terms of age $(41.32 \pm 2.234 \mathrm{vs}$. $36.63 \pm 1.821, p=0.11$ ) or body mass index (BMI; $24.88 \pm 0.7089$ vs. $23.96 \pm 0.8668, p=0.45)$. According to Rome II sub classification of predominant bowel habit, nine had constipationpredominant IBS (IBS-C), eight had diarrhea-predominant IBS (IBS-D), and eight had alternating IBS (IBS-A). There were three current smokers in each group. Eleven IBS subjects (44\%) met criteria for a current psychiatric co-morbidity. According to IBS symptom severity scores, 5 patients rated their symptoms as mild, 12 as moderate and 8 as severe.

\section{PLASMA TRYPTOPHAN AND KYNURENINE CONCENTRATIONS}

There was no significant difference in plasma tryptophan levels between controls and IBS groups $(10.0 \pm 0.4$ vs. $\left.10.5 \pm 0.7 \mu \mathrm{g} \mathrm{mL}^{-1}, p=0.51\right)$. Plasma kynurenine levels were significantly elevated in comparison to controls levels in IBS patients $\left(0.39 \pm 0.02\right.$ vs. $\left.0.50 \pm 0.05 \mu \mathrm{g} \mathrm{mL}^{-1}, p<0.05\right)$ as was the kynurenine:tryptophan ratio $(0.03991 \pm 0.00222$ vs. $0.04795 \pm 0.00315$, $p<0.05$ ), an index of IDO activity (Figures 1A-C).

\section{TLR AGONIST INDUCED ALTERNATIONS IN TRYPTOPHAN DEGRADATION.}

Concentrations of tryptophan and kynurenine following TLR stimulation are given in Tables 1 and 2 respectively.

Two-way ANOVA analysis revealed a significant interaction between disease state and treatment $\left(F_{3,126}=5.867, p<0.05\right)$ for the kynurenine:tryptophan ratio following stimulation of whole blood with the TLR1/2 agonist Pam3Csk. Post hoc analysis indicated a significant reduction in this ratio, in the IBS group, in stimulated preparations compared to the unstimulated samples $(0.01774 \pm 0.00089$ vs. $0.01493 \pm 0.00098, p<0.05)$ with no alteration in this ratio in the samples taken from the healthy controls (Figure 2A). Two-way ANOVA analysis revealed a significant interaction between disease state and treatment $\left(F_{3,126}=8.475\right.$, $p<0.01$ ) following stimulation of whole blood with the TLR2 agonist HKLM. Post hoc analysis revealed a significant increase in this ratio in the healthy control group in stimulated vs. unstimulated samples $(0.01681 \pm 0.0006$ vs. $0.02159 \pm 0.00098, p<0.001)$ with no alteration in this ratio in the samples taken from IBS patients (Figure 2B). There was a trend toward a significant interaction between disease state and treatment $\left(F_{3,126}=2.887\right.$, $p=0.092$ ) following stimulation with the TLR3 agonist Poly I:C. Post hoc analysis indicated a significant increase in the ratio in the healthy control group in stimulated vs. unstimulated samples $(0.01681 \pm 0.0006$ vs. $0.01907 \pm 0.00092, p<0.05)$ with no alteration in the ratio being demonstrated in the samples taken from IBS patients (Figure 2C).

There was no significant interaction between disease state and treatment following stimulation with the TLR4 agonist LPS $\left(F_{3,125}=0.157, p=0.962\right)$. Post hoc analysis indicated a significant increase in the ratio in the stimulated compared to unstimulated samples from both controls $(0.01681 \pm 0.0006$ vs. $0.02354 \pm 0.00112, p<0.001)$ and IBS patients $(0.01774 \pm 0.00089$ vs. $0.02367 \pm 0.00137, p<0.001$; Figure $3 \mathbf{A})$. There was a trend toward a significant interaction between disease state and treatment following stimulation with the TLR5 agonist flagellin $\left(F_{3,124}=3.604, p=0.06\right)$. The kynurenine:tryptophan ratio was significantly elevated from unstimulated levels only in the samples taken from IBS patients $(0.01774 \pm 0.00089$ vs. $0.02259 \pm 0.0015, p<0.01$; Figure 3B).

There was no significant interaction between disease state and treatment following stimulation with the TL6/2 agonist FSL1 $\left(F_{1,124}=1.026, p=0.313\right)$ not was there any alteration in the kynurenine:tryptophan ratio in either healthy controls or IBS subjects (Figure 3C).

There was no significant interaction between disease state and treatment following stimulation with the TLR7 agonist imiquimod $\left(F_{3,125}=0.146, p=0.703\right)$ although this agonist did induce an increase in the kynurenine:tryptophan ratio in stimulated samples from control subjects $(0.01681 \pm 0.0006$ vs. $0.01954 \pm 0.00087, p<0.05)$ that was not evident in their IBS counterparts (Figure 4A). There was a trend toward a significant interaction between disease state and treatment following stimulation of samples with the TLR8 agonist ssRNA40 $\left(F_{3,124}=3.491, p=0.064\right)$. This TLR ligand induced an increase in the kynurenine:tryptophan ratio only in the stimulated samples from IBS patients $(0.01774 \pm 0.00089$ vs. $0.02166 \pm 0.00161$, $p<0.05$; Figure 4B). There was no significant interaction between disease state and treatment following stimulation with the TLR9 agonist ODN2006 $\left(F_{3,125}=2.324, p=0.131\right)$ nor was there any alteration in the kynurenine:tryptophan ratio in the stimulated samples from either healthy control or IBS subjects (Figure 4C). 

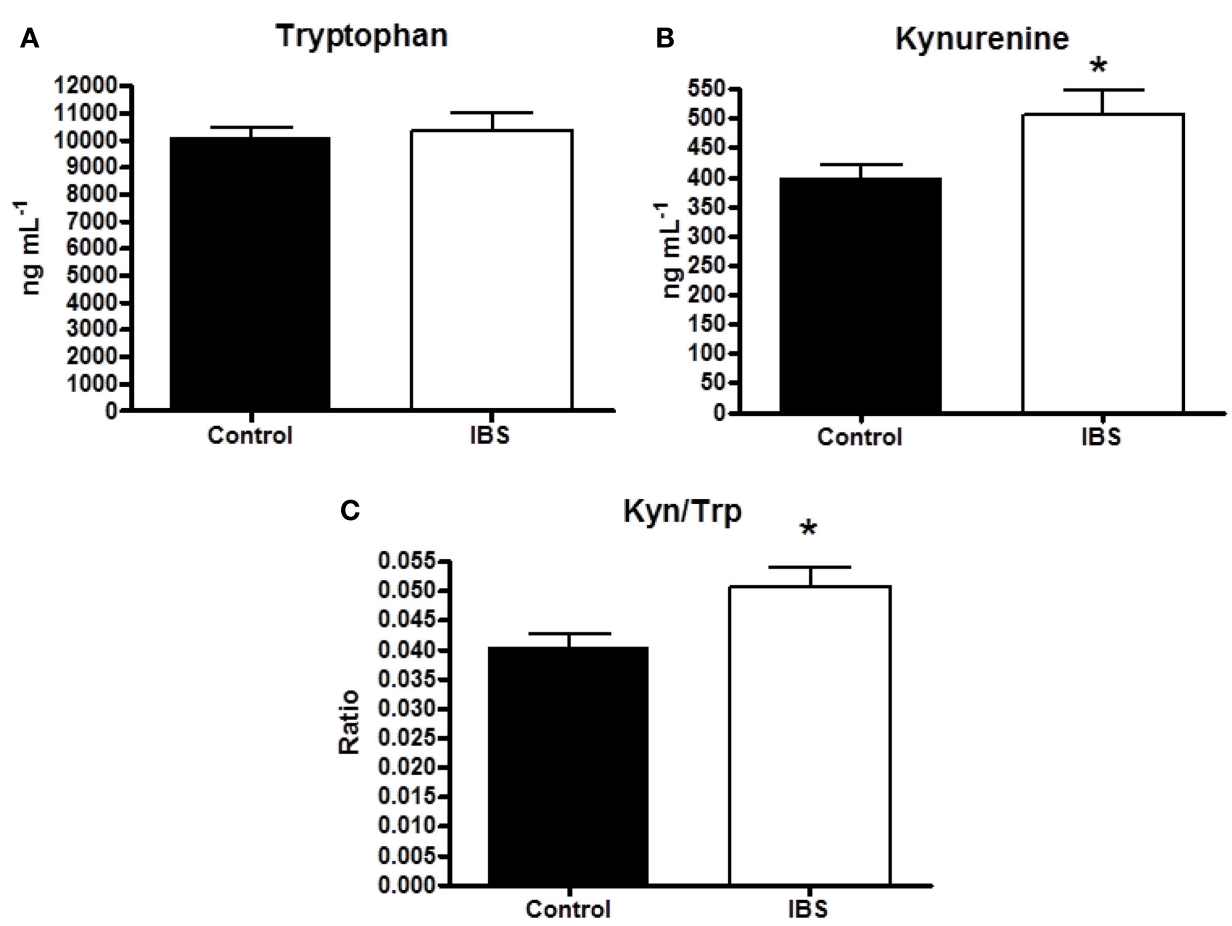

FIGURE 1 | (A) Plasma tryptophan concentrations ( $\mu \mathrm{g} \mathrm{mL}^{-1}$ ) in healthy controls and IBS patients. (B) Plasma kynurenine concentrations ( $\mu \mathrm{g} \mathrm{mL}^{-1}$ ) in healthy controls and IBS patients. (C) Plasma kynurenine:tryptophan
(Kyn:Tryp) ratio in healthy controls and IBS patients. Data are expressed as means \pm SEM. Statistical differences between healthy controls and IBS patients were determined using Student's $t$-test. ${ }^{*} p<0.05$.

Table 1 | Tryptophan concentrations $\left(\mu \mathrm{g} \mathrm{mL}^{-1}\right)$ in unstimulated and TLR agonist stimulated whole blood supernatants from healthy controls and IBS patients.

Control

\begin{tabular}{llllll}
\hline TLR stimulation & Tryptophan & $\boldsymbol{p}$ & & Tryptophan & $\boldsymbol{p}$ \\
\hline Unstimulated & $13.5 \pm 0.2$ & $\mathrm{~N} / \mathrm{A}$ & & $13.7 \pm 0.3$ & $\mathrm{~N} / \mathrm{A}$ \\
1/2 (Pam3Cys) & $11.7 \pm 0.2$ & $* * *$ & & $12.0 \pm 0.3$ & $\$ \$$ \\
2 (HKLM) & $13.1 \pm 0.3$ & - & $12.1 \pm 0.3$ & $\$ \$$ \\
3 (Poly I:C) & $11.8 \pm 0.3$ & $* * *$ & $12.3 \pm 0.2$ & $\$ \$$ \\
4 (LPS) & $13.7 \pm 0.4$ & - & $13.7 \pm 0.4$ & - \\
5 (Flagellin) & $13.3 \pm 0.3$ & - & $13.3 \pm 0.3$ & - \\
6/2 (FSL1) & $12.6 \pm 0.2$ & $*$ & & $13.2 \pm 0.3$ & - \\
7 (Imiquimod) & $13.0 \pm 0.2$ & - & $13.0 \pm 0.2$ & - \\
8 (ssRNA40) & $12.6 \pm 0.3$ & $*$ & & $12.9 \pm 0.2$ & - \\
9 (ODN2006) & $13.4 \pm 0.5$ & - & & $13.0 \pm 0.5$ & -
\end{tabular}

Data are expressed as means \pm SEM. Statistical differences between stimulated and unstimulated groups were determined using Bonferroni post hoc test. ${ }^{*} p<0.05$, Healthy control unstimulated $v s$. TLR agonist stimulated; ${ }^{* *} p<0.001$, healthy control unstimulated vs. TLR agonist Stimulated; $\$ \$ p<0.01$, IBS unstimulated vs. stimulated; $-p>0.05$.

\section{DISCUSSION}

Previous reports from our laboratory have indicated increased degradation of tryptophan along the kynurenine pathway and highlighted the potential utility of these indices as biological
Table 2 | Kynurenine concentrations $\left(\mu \mathrm{g} \mathrm{mL}^{-1}\right)$ in unstimulated and TLR agonist stimulated whole blood supernatants from healthy controls and IBS patients.

\begin{tabular}{|c|c|c|c|c|}
\hline \multicolumn{3}{|c|}{ Control } & \multicolumn{2}{|l|}{ IBS } \\
\hline TLR stimulation & Kynurenine & $p$ & Kynurenine & $p$ \\
\hline Unstimulated & $0.22 \pm 0.01$ & $\mathrm{~N} / \mathrm{A}$ & $0.24 \pm 0.01$ & \\
\hline 1/2 (Pam3Cys) & $0.21 \pm 0.01$ & - & $0.18 \pm 0.01$ & $\$ \$ \$$ \\
\hline 2 (HKLM) & $0.27 \pm 0.01$ & $* * *$ & $0.20 \pm 0.01$ & $\$$ \\
\hline 3 (Poly I:C) & $0.22 \pm 0.01$ & - & $0.19 \pm 0.01$ & $\$ \$$ \\
\hline 4 (LPS) & $0.33 \pm 0.01$ & $* * *$ & $0.32 \pm 0.02$ & $\$ \$ \$$ \\
\hline 5 (Flagellin) & $0.23 \pm 0.01$ & - & $0.30 \pm 0.02$ & $\$ \$$ \\
\hline 6/2 (FSL1) & $0.21 \pm 0.01$ & - & $0.21 \pm 0.01$ & - \\
\hline 7 (Imiquimod) & $0.25 \pm 0.01$ & - & $0.26 \pm 0.02$ & - \\
\hline 8 (ssRNA40) & $0.23 \pm 0.01$ & - & $0.28 \pm 0.02$ & - \\
\hline 9 (ODN2006) & $0.25 \pm 0.01$ & & $0.21 \pm 0.01$ & - \\
\hline
\end{tabular}

Data are expressed as means \pm SEM. Statistical differences between stimulated and unstimulated groups were determined using Bonferroni post hoc test. ${ }^{* * *} p<0.001$, Healthy control unstimulated vs. TLR agonist stimulated; $\$ p<0.05$, IBS unstimulated vs. TLR agonist stimulated; $\$ \$<0.01$, IBS unstimulated vs. stimulated; $\$ \$ \$ p<0.001$ IBS unstimulated vs. TLR agonist stimulated; $p>0.05$.

markers of IBS (Fitzgerald et al., 2008; Clarke et al., 2009a). Here we have confirmed these findings by demonstrating increased plasma kynurenine concentrations as well as an elevation in the 


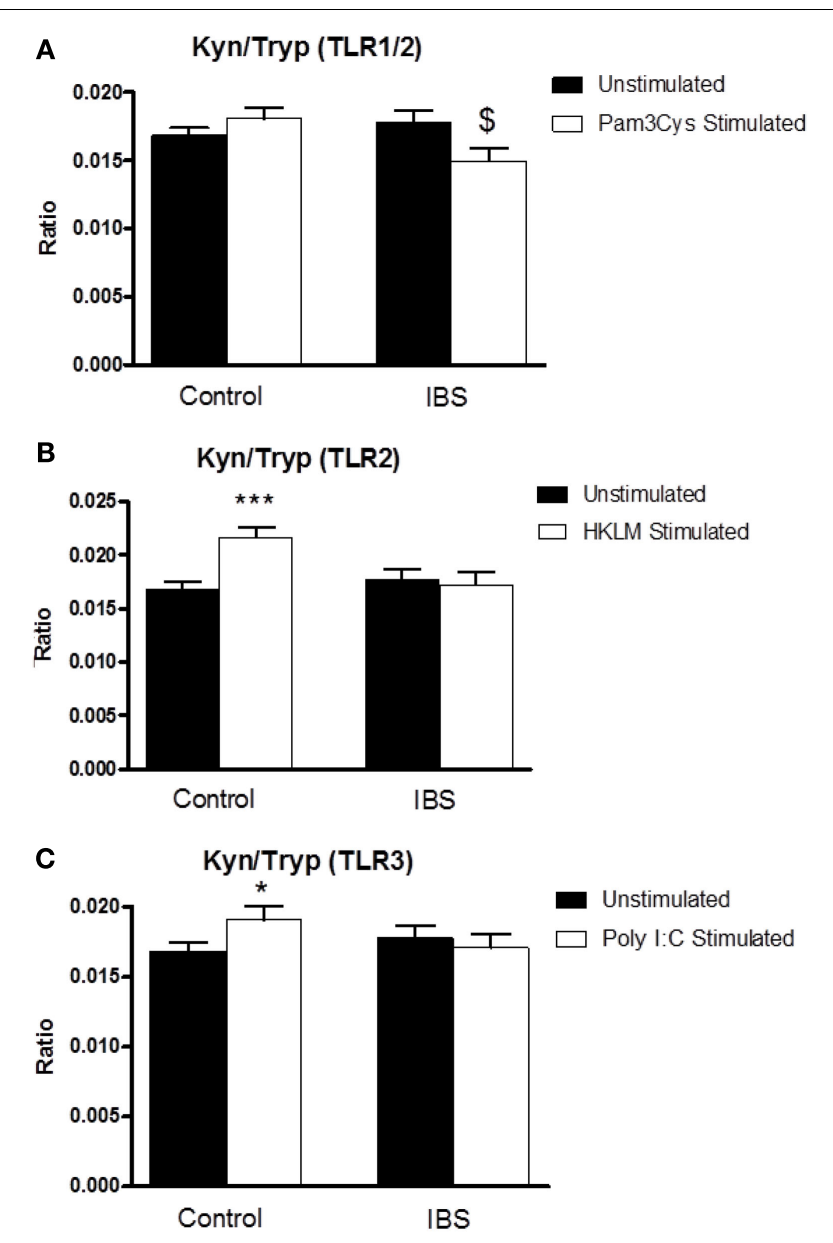

FIGURE 2 | Kynurenine:tryptophan (Kyn:Tryp) ratio in unstimulated whole blood supernatants from healthy controls and IBS patients and following stimulation with: (A) TLR1/2 agonist Pam3Csk, (B) TLR2 agonist HKLM, and (C) TLR3 agonist Poly I:C. Data are expressed as mean \pm SEM. Statistical differences between treatments and disease states were determined using two-way ANOVA with Bonferroni post hoc test. ${ }^{*} p<0.05$, Healthy control stimulated vs. unstimulated; ${ }^{*}{ }^{*} p<0.001$, healthy control stimulated vs. unstimulated; $\$ p<0.05$, IBS stimulated vs. unstimulated.

kynurenine:tryptophan ratio in our IBS cohort compared to controls. Moreover, we have demonstrated, for the first time, that IBS patients exhibit a distinct tryptophan degradation profile downstream of TLR activation that is different from that of healthy controls, as indicated by the kynurenine:tryptophan ratio in the supernatants of whole blood preparations.

The pathophysiological relevance of increased activity along the kynurenine pathway in the plasma of IBS patients remains to be defined as does the source of such alterations. Of the TLRs that have previously been demonstrated to be upregulated in the colon in IBS (Brint et al., 2011) and to have an elevated cytokine release profile (McKernan et al., 2011), both TLR5 and TLR8 induced, on activation, an increase in the kynurenine:tryptophan ratio in our experimental system. It has recently been shown that alterations in gastrointestinal IDO activity in Crohns's disease correlate with systemic indices of kynurenine pathway activation (Gupta et al.,

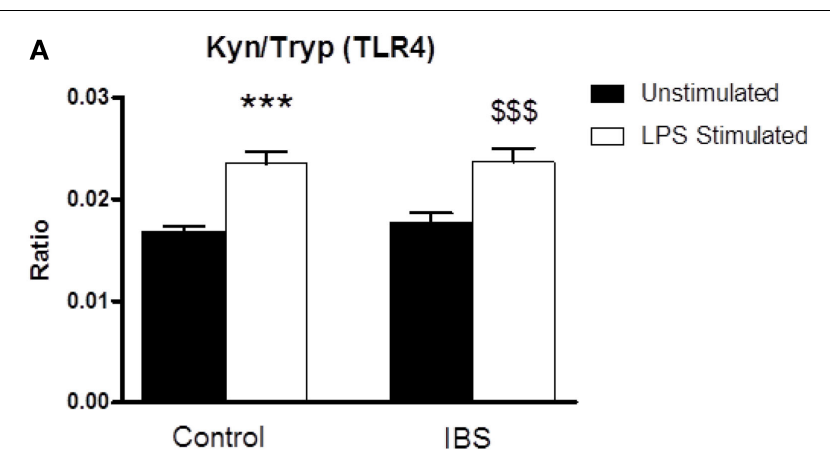

B Kyn/Tryp (TLR5)

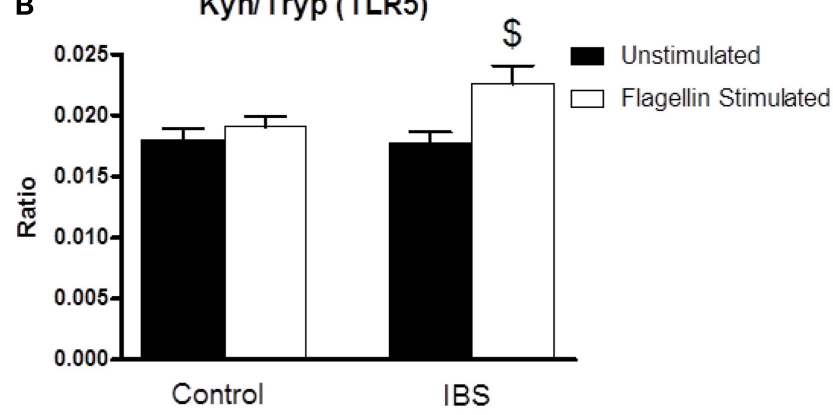

C

Kyn/Tryp (TLR6)

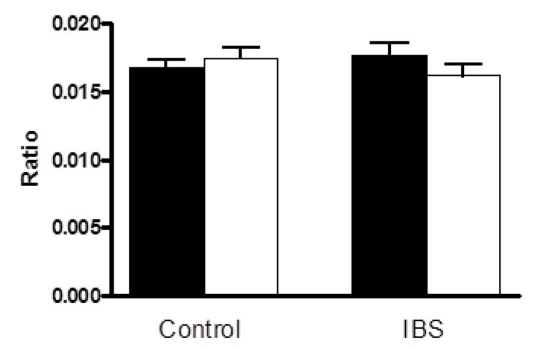

Unstimulated $\square$ FSL1 Stimulated

FIGURE 3 | Kynurenine:tryptophan (Kyn:Tryp) ratio in unstimulated whole blood supernatants from healthy controls and IBS patients and following stimulation with: (A) TLR4 agonist LPS, (B) TLR5 agonist flagellin, and (C) TLR6/1 agonist FSL1. Data are expressed as mean \pm SEM. Statistical differences between treatments and disease states were determined using two-way ANOVA with Bonferroni post hoc test. ${ }^{* *} p<0.001$, Healthy control stimulated vs. unstimulated; $\$ p<0.05$, IBS stimulated vs. unstimulated; $\$ \$ \$<0.001$, IBS stimulated vs. unstimulated. There was no significant interaction between disease state and treatment following stimulation with the TL6/2 agonist FSL1

$\left(F_{1,124}=1.026, p=0.313\right)$ not was there any alteration in the kynurenine:tryptophan ratio in either healthy controls or IBS subjects (Figure 3C).

2011). Moreover, it is becoming increasingly apparent that peripheral blood alterations in kynurenine pathway metabolites can manifest at the CNS level (Raison et al., 2009) and that modulation of systemic pathway activity might be a useful therapeutic strategy (Reinhart and Kelly, 2011; Zwilling et al., 2011). Nevertheless, it will be important to determine whether the findings presented here are indeed reflected at the level of the intestinal compartment, an important site of tryptophan metabolism and serotonergic signaling in the periphery and further studies using supernatants from mucosal biopsies will be of value in this regard. 


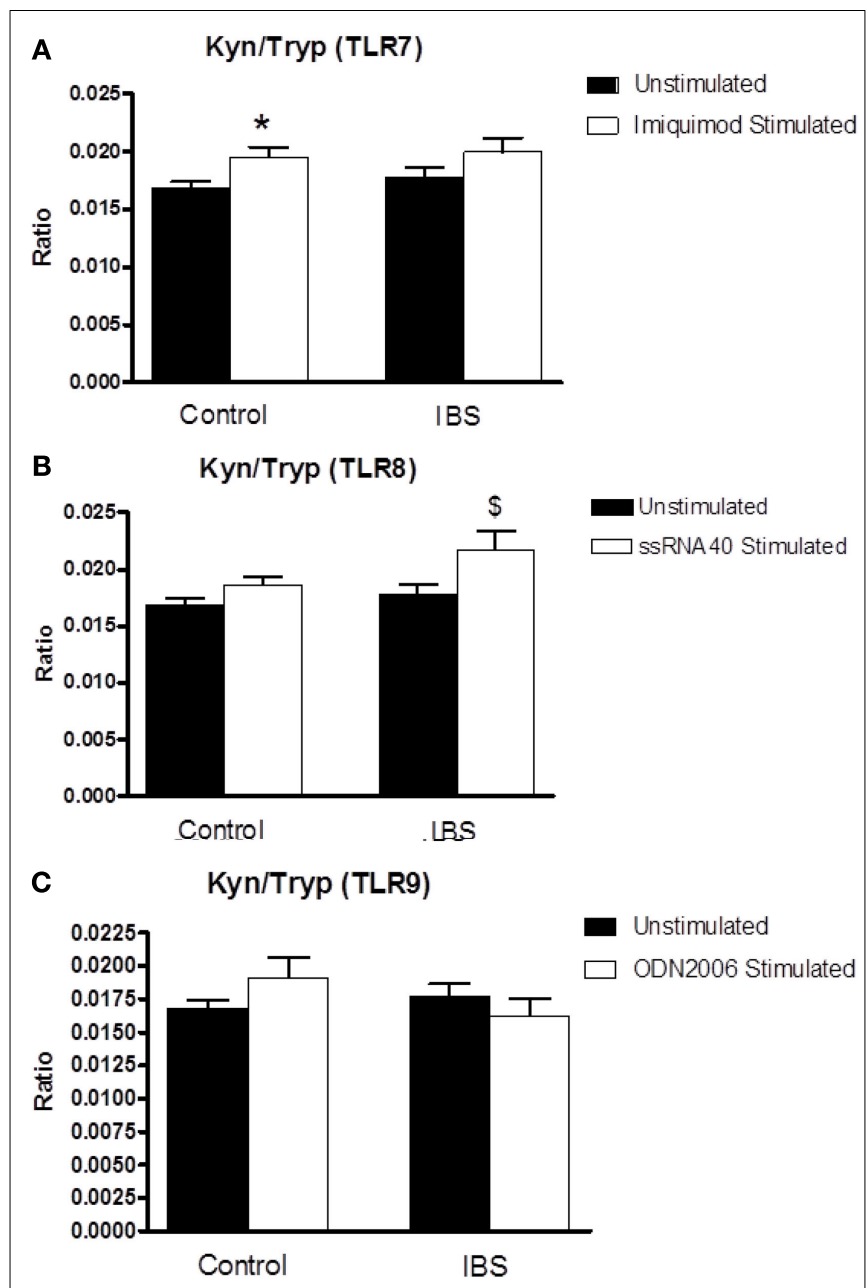

FIGURE 4 | Kynurenine:tryptophan (Kyn:Tryp) ratio in unstimulated whole blood supernatants from healthy controls and IBS patients and following stimulation with: (A) TLR 7 agonist Imiquimod, (B) TLR8 agonist ssRNA40, and (C) TLR9 agoinst ODN2006. Data are expressed as mean \pm SEM. Statistical differences between treatments and disease states were determined using two-way ANOVA with Bonferroni post hoc test. ${ }^{*} p<0.05$, Healthy control stimulated vs. unstimulated; $\$ p<0.05$, IBS stimulated vs. unstimulated.

The differential tryptophan degradation profile at TLR1/2, TLR2, and TLR3 is an important finding. These receptors are expressed at both intracellular and extracellular domains (Akira and Takeda, 2004; Sioud, 2006) and our data suggests the presence, in IBS, of TLR dysfunction at the level of both the cell membrane and the endosome. It is also noteworthy that TLRs can interact at a functional level to limit or inhibit the normal response to a particular ligand (Hajjar et al., 2001), a feature which may be of relevance to the reduced kynurenine:tryptophan ratio observed in IBS patients following stimulation with the TLR1/2 ligand. An alternative explanation, given that our ratio alterations following stimulation of TLR1/2 and TLR3 are derived from reductions in tryptophan concentrations in conjunction with decreased kynurenine, may involve the sequestering of tryptophan for the diverse cellular processes in which it is involved. Certain cytokines, for example, can upregulate tryptophan hydroxylase expression, the rate-limiting enzyme in the conversion of tryptophan to serotonin (Lisak et al., 2011). Future studies will also need to address whether a differential leukocyte distribution might account for our findings although recent studies suggest that cellular activation might be a more important factor (Ohman et al., 2009a,b).

In any case, the blunted kynurenine production at TLR2 and TLR3 in our IBS cohort is perplexing given the enhanced production of TNF- $\alpha$ and IL- 8 demonstrated at those receptor subtypes, respectively, in our earlier study (McKernan et al., 2011) and the recently reported increased TLR2 expression on blood monocytes in IBS patients (Ohman et al., 2012). It is, however, worth noting that combinations of elevated cytokines may favour kynurenine production over the singular increases described at these receptor subtypes (Taylor and Feng, 1991) and that the availability of certain co-factors is also required (Muller and Schwarz, 2007). Additionally, the spontaneous release of IL- 8 from unstimulated samples, a previously reported phenomenon (Molina et al., 2006; Horton and Remick, 2010), from both groups and the baseline differences in the concentration of this cytokine may have obscured the impact that post-stimulation alterations in IL- 8 production may have produced. Also of relevance is that repeated stimulation of specific TLRs with their ligands can induce unresponsiveness or immunotolerance, at least in cell line studies (Ehlers and Ravetch, 2007; Gomez-Llorente et al., 2010). This raises the possibility that the defective response at TLR2 and TLR3 in IBS subjects might alternatively be due to prolonged stimulation by endogenous ligands. This is in line with suggestions that activation of TLRs by their endogenous ligands might have a role in the promotion of systemic inflammation (Marshak-Rothstein, 2006). Interestingly host mRNA can activate TLR3 (Kariko et al., 2004) and high mobility group box 1 (HMGB1) is a ligand for TLR2 (Yu et al., 2006). Total plasma mRNA levels remain to be profiled in IBS and although fecal HMGB1 has been proposed as a novel marker of intestinal inflammation, it is also uncharacterised in the disorder.

Interestingly, we found that the downstream consequences of TLR4 activation for tryptophan metabolism are equivalent in both healthy controls and IBS patients. Previously we reported an increased release of the same two cytokines (IL-1B, TNF- $\alpha$ ) in IBS patients following stimulation of this receptor with LPS as occurred following TLR5 activation with flagellin (McKernan et al., 2011). At first glance, it appears unusual that an exaggerated release of the same two cytokines would elicit differential responses in terms of kynurenine pathway indices at different TLRs if the immunoresponsive enzyme IDO mediates the effects we have observed. However, it should be noted that the magnitude of the cytokine response following TLR4 stimulation is greater than that induced following TLR5 activation suggesting that the more modest cytokine release pattern at the latter receptor may be required to tease apart the differential downstream effects of TLR stimulation on kynurenine production. The cellular distribution of the TLRs may also be of importance: it is worth noting that TLR4 is much more highly expressed than TLR5 in monocytes (Hornung et al., 2002) and LPS stimulation of whole blood primarily results in cytokine release from this cell type (Pace and Heim, 2011). In contrast, TLR5 is more highly expressed in NK cells and T cells 
than TLR4, although the magnitude of dominance TLR5 enjoys in these cell populations does not match the superiority of TLR4 in terms of monocyte expression patterns. Although we did not analyze the cellular composition of our whole blood preparations, this theory is consistent with the observation that IBS subjects display an increased level of T-cell activation (Ohman et al., 2009a). This explanation also reflects the view in the literature that IBS is a disorder characterised by a low grade inflammation as opposed to an immune disorder per se (Clarke et al., 2009b). However it should also be noted that TLR4 stimulation increased kynurenine concentrations without altering tryptophan concentrations which may suggest the involvement of enzymatic components of the kynurenine pathway other than IDO. Indeed differential activation of kynurenine pathway enzymes can be a feature of a systemic immune challenge with LPS (Connor et al., 2008).

A limitation of the current study is that whole blood stimulations of TLRs may only partially model the complexity of the in vivo response. This is especially pertinent when one considers that the entire family of receptors may be simultaneously susceptible to activation in vivo depending on the combination of ligands present at physiologically relevant concentrations. Indeed, it is known that combinations of TLR ligands can either synergistically induce cytokine gene expression (Makela et al., 2011) or result in a blunted response (Marshall et al., 2007), depending on the particular ligand combination employed. Consequently, our results do not clarify whether the cumulative, sometimes opposing and sometimes synergistic, downstream effects of multiple simultaneous TLR activations are responsible for the enhanced resting state degradation of tryptophan along the kynurenine pathway in IBS. Moreover, it is unclear at present which receptor has the greatest biological influence on kynurenine metabolism in vivo. Nevertheless whole blood stimulation of single receptor subtypes is considered a valid strategy to interrogate their responsiveness in general and to determine whether particular patient populations are primed for aberrant responses (Pace and Heim, 2011). Moreover, an assessment of the kynurenine:tryptophan ratio is a previously validated method for the assessment of IDO activity in

\section{REFERENCES}

Akira, S., and Takeda, K. (2004). Toll-like receptor signalling. NatRev Immunol. 4, 499-511.

Bercik, P., Collins, S. M., and Verdu, E. F. (2012). Microbes and the gutbrain axis. Neurogastroenterol. Motil. 24, 405-413.

Brint, E. K., Macsharry, J., Fanning, A., Shanahan, F., and Quigley, E. M. (2011). Differential expression of toll-like receptors in patients with irritable bowel syndrome. Am. J. Gastroenterol. 106, 329-336.

Clarke, G., Fitzgerald, P., Cryan, J. F., Cassidy, E. M., Quigley, E. M., and Dinan, T. G. (2009a). Tryptophan degradation in irritable bowel syndrome: evidence of indoleamine 2,3dioxygenase activation in a male cohort. BMC Gastroenterol. 9, 6. doi:10.1186/1471-230X-9-6
Clarke, G., Fitzgerald, P., Hennessy, A. A., Cassidy, E. M., Quigley, E. M. Ross, P., Stanton, C., Cryan, J. F., and Dinan, T. G. (2010). Marked elevations in pro-inflammatory polyunsaturated fatty acid metabolites in females with irritable bowel syndrome. J. Lipid Res. 51, 1186-1192.

Clarke, G., Quigley, E. M., Cryan, J. F., and Dinan, T. G. (2009b). Irritable bowel syndrome: towards biomarker identification. Trends. Mol. Med. 15, 478-489.

Connor, T. J., Starr, N., O’Sullivan, J. B., and Harkin, A. (2008). Induction of indolamine 2,3-dioxygenase and kynurenine 3-monooxygenase in rat brain following a systemic inflammatory challenge: a role for IFNgamma? Neurosci. Lett. 441, 29-34.

Cremon, C., Gargano, L., MorselliLabate, A. M., Santini, D., Cogliandro, R. F., De Giorgio,

cell culture supernatants (Schroecksnadel et al., 2005; Mahanonda et al., 2007; Schroecksnadel et al., 2011). The fact that IBS populations show both basal alterations in this pathway and a distinct profile subsequent to stimulation of individual TLRs with their various ligands only adds to their potential utility as biomarkers of the disorder, albeit as components of a biomarker panel of other promising indices rather than as unique identifiers themselves. Further studies are urgently required, both to define such a biomarker panel and to determine the sensitivity and specificity of such an approach.

In conclusion, we have provided novel evidence demonstrating that TLR activation induces a pattern of downstream tryptophan degradation along the kynurenine pathway that differentiates IBS patients from healthy controls. This bolsters current and previous findings that highlighted baseline disturbances in this pathway and illuminates a mechanism through which TLR responses can functionally impact on brain-gut axis signaling in this disorder. Moreover, this implicates the modulation of TLRs as a novel therapeutic strategy in this debilitating condition.

\section{ACKNOWLEDGMENTS}

Timothy G. Dinan, John F. Cryan, and Eamonn M. Quigley designed the study. Eamonn M. Quigley, Declan P. McKernan, and Gabor Gaszner coordinated subject recruitment. Declan P. McKernan, Gabor Gaszner, and Gerard Clarke carried out the research. Gerard Clarke analyzed the data, interpreted the results and wrote the paper. The Alimentary Pharmabiotic Centre is a research centre funded by Science Foundation Ireland (SFI), through the Irish Government's National Development Plan. The authors and their work were supported by SFI (grant nos. 02/CE/B124 and 07/CE/B1368) and by GlaxoSmithKline. Gerard Clarke is in receipt of a research grant from the American Neurogastroenterology and Motility Society (ANMS). Timothy G. Dinan, John F. Cryan and Gerard Clarke are also supported by the Health Research Board (HRB) through Health Research Awards (grant no HRA_POR/2011/23).

R., Stanghellini, V., Corinaldesi, R., and Barbara, G. (2009). Mucosal immune activation in irritable bowel syndrome: gender-dependence and association with digestive symptoms. Am. J. Gastroenterol. 104, 392-400.

Crowell, M. D. (2004). Role of serotonin in the pathophysiology of the irritable bowel syndrome. Br. J. Pharmacol. 141, 1285-1293.

Dantzer, R., O’Connor, J. C., Freund, G. G., Johnson, R. W., and Kelley, K. W. (2008). From inflammation to sickness and depression: when the immune system subjugates the brain. Nat. Rev. Neurosci. 9, 46-56.

Dinan, T. G., Clarke, G., Quigley, E. M., Scott, L. V., Shanahan, F., Cryan, J., Cooney, J., and Keeling, P. W. (2008). Enhanced cholinergic-mediated increase in the pro-inflammatory cytokine IL-6 in irritable bowel syndrome: role of muscarinic receptors. Am. J. Gastroenterol. 103, 2570-2576.

Dinan, T. G., Quigley, E. M., Ahmed, S. M., Scully, P., O'Bbrien, S., O'Mahony, L., O’Mahony, S., Shanahan, F., and Keeling, P. W. (2006). Hypothalamicpituitary-gut axis dysregulation in irritable bowel syndrome: plasma cytokines as a potential biomarker? Gastroenterology 130, 304-311.

Drossman, D. A., and Dumitrascu, D. L. (2006). Rome III: new standard for functional gastrointestinal disorders. J. Gastrointestin. Liver Dis. 15, 237-241.

Ehlers, M., and Ravetch, J. V. (2007). Opposing effects of Toll-like receptor stimulation induce autoimmunity or tolerance. Trends Immunol. 28, 74-79. 
Fitzgerald, P., Cassidy Eugene, M., Clarke, G., Scully, P., Barry, S., Quigley Eamonn, M. M., Shanahan, F., Cryan, J., and Dinan Timothy, G. (2008). Tryptophan catabolism in females with irritable bowel syndrome: relationship to interferon-gamma, severity of symptoms and psychiatric co-morbidity. Neurogastroenterol. Motil. 20, 1291-1297.

Forsythe, P., Sudo, N., Dinan, T., Taylor, V. H., and Bienenstock, J. (2010). Mood and gut feelings. Brain Behav. Immun. 24, 9-16.

Francis, C. Y., Morris, J., and Whorwell, P. J. (1997). The irritable bowel severity scoring system: a simple method of monitoring irritable bowel syndrome and its progress. Aliment. Pharmacol. Ther. $11,395-402$

Gomez-Llorente, C., Munoz, S., and Gil, A. (2010). Role of toll-like receptors in the development of immunotolerance mediated by probiotics. Proc. Nutr. Soc. 69, 381-389.

Grenham, S., Clarke, G., Cryan, J. F., and Dinan, T. G. (2011). Brain-gutmicrobe communication in health and disease. Front. Physiol. 2:94. doi:10.3389/fphys.2011.00094

Gupta, N. K., Thaker, A. I., Kanuri, N., Riehl, T. E., Rowley, C. W., Stenson, W. F., and Ciorba, M. A. (2011). Serum analysis of tryptophan catabolism pathway: correlation with Crohn's disease activity. Inflamm. Bowel Dis. doi:10.1002/ibd.21849

Hajjar, A. M., O'Mahony, D. S., Ozinsky, A., Underhill, D. M., Aderem, A., Klebanoff, S. J., and Wilson, C. B. (2001). Cutting edge: functional interactions between tolllike receptor (TLR) 2 and TLR1 or TLR6 in response to phenolsoluble modulin. J. Immunol. 166, 15-19.

Herve, C., Beyne, P., Jamault, H., and Delacoux, E. (1996). Determination of tryptophan and its kynurenine pathway metabolites in human serum by high-performance liquid chromatography with simultaneous ultraviolet and fluorimetric detection. J. Chromatogr. B Biomed. Appl. 675, 157-161.

Hornung, V., Rothenfusser, S., Britsch, S., Krug, A., Jahrsdorfer, B., Giese, T., Endres, S., and Hartmann, G. (2002). Quantitative expression of toll-like receptor 1-10 mRNA in cellular subsets of human peripheral blood mononuclear cells and sensitivity to $\mathrm{CpG}$ oligodeoxynucleotides. $J$. Immunol. 168, 4531-4537.

Horton, D. L., and Remick, D. G. (2010). Delayed addition of glucocorticoids selectively suppresses cytokine production in stimulated human whole blood. Clin. Vaccine Immunol. 17, 979-985.

Kariko, K., Ni, H., Capodici, J., Lamphier, M., and Weissman, D. (2004). mRNA is an endogenous ligand for Toll-like receptor 3. J. Biol. Chem. 279, 12542-12550.

Kennedy, P. J., Clarke, G., Quigley, E. M., Groeger, J. A., Dinan, T. G., and Cryan, J. F. (2012). Gut memories: towards a cognitive neurobiology of irritable bowel syndrome. Neurosci. Biobehav. Rev. 36, 310-340.

Liebregts, T., Adam, B., Bredack, C., Roth, A., Heinzel, S., Lester, S., Downie-Doyle, S., Smith, E., Drew, P., Talley, N. J., and Holtmann, G. (2007). Immune activation in patients with irritable bowel syndrome. Gastroenterology 132, 913-920.

Lisak, R. P., Nedelkoska, L., Studzinski, D., Bealmear, B., Xu, W., and Benjamins, J. A. (2011). Cytokines regulate neuronal gene expression: differential effects of Th1, Th2 and monocyte/macrophage cytokines. J. Neuroimmunol. 238, 19-33.

Mahanonda, R., Sa-Ard-Iam, N., Montreekachon, P., Pimkhaokham, A., Yongvanichit, K., Fukuda, M. M., and Pichyangkul, S. (2007). IL-8 and IDO expression by human gingival fibroblasts via TLRs. J. Immunol. 178, 1151-1157.

Makela, S. M., Osterlund, P., and Julkunen, I. (2011). TLR ligands induce synergistic interferon-beta and interferon-lambdal gene expression in human monocytederived dendritic cells. Mol. Immunol. 48, 505-515.

Marshak-Rothstein, A. (2006). Tolllike receptors in systemic autoimmune disease. Nat. Rev. Immunol. 6 , 823-835.

Marshall, J. D., Heeke, D. S., Gesner, M. L., Livingston, B., and Van Nest, G. (2007). Negative regulation of TLR9-mediated IFN-alpha induction by a small-molecule, synthetic TLR7 ligand. J. Leukoc. Biol. 82, 497-508.

Mayer, E. A. (2011). Gut feelings: the emerging biology of gut-brain communication. Nat. Rev. Neurosci. 12, 453-466.

McKernan, D. P., Gaszner, G., Quigley, E. M., Cryan, J. F., and Dinan, T. G. (2011). Altered peripheral toll-like receptor responses in the irritable bowel syndrome. Aliment. Pharmacol. Ther. 33, 1045-1052.

McKernan, D. P., Nolan, A., Brint, E. K., O'Mahony, S. M., Hyland, N.
P., Cryan, J. F., and Dinan, T. G. (2009). Toll-like receptor mRNA expression is selectively increased in the colonic mucosa of two animal models relevant to irritable bowel syndrome. PLoS ONE 4, e8226. doi:10.1371/journal.pone.0008226

Molina, M. A., Gamboa, E. M., Tello, P. C., Benavides, P. Z., Leon, L. C., Guerra, R. T., and Padilla, C. R. (2006). Spontaneous inflammatory cytokine gene expression in normal human peripheral blood mononuclear cells. Lymphat. Res. Biol. 4 34-40.

Muller, N., and Schwarz, M. J. (2007). The immune-mediated alteration of serotonin and glutamate: towards an integrated view of depression. Mol. Psychiatry 12, 988-1000.

O'Malley, D., Quigley, E. M., Dinan, T. G., and Cryan, J. F. (2011). Do interactions between stress and immune responses lead to symptom exacerbations in irritable bowel syndrome? Brain Behav. Immun. 25, 1333-1341.

Ohman, L., Isaksson, S., Lindmark, A. C., Posserud, I., Stotzer, P. O., Strid, H., Sjovall, H., and Simren, M. (2009a). T-cell activation in patients with irritable bowel syndrome. Am J. Gastroenterol. 104, 1205-1212.

Ohman, L., Lindmark, A. C., Isaksson, S., Posserud, I., Strid, H., Sjovall, H., and Simren, M. (2009b). B-cell activation in patients with irritable bowel syndrome (IBS). Neurogastroenterol. Motil. 21, 644-650.

Ohman, L., Lindmark, A. C., Isaksson, S., Posserud, I., Strid, H., Sjovall, H. and Simren, M. (2012). Increased TLR2 expression on blood monocytes in irritable bowel syndrome patients. Eur. J. Gastroenterol. Hepatol. 24, 398-405.

Ohman, L., and Simren, M. (2007) New insights into the pathogenesis and pathophysiology of irritable bowel syndrome. Dig. Liver Dis. 39, 201-215.

Ohman, L., and Simren, M. (2010). Pathogenesis of IBS: role of inflammation, immunity and neuroimmune interactions. Nat. Rev. Gas troenterol. Hepatol. 7, 163-173.

Pace, T. W., and Heim, C. M. (2011). A short review on the psychoneuroimmunology of posttraumatic stress disorder: from risk factors to medical comorbidities. Brain Behav. Immun. 25, 6-13.

Quigley, E. M. (2006). Changing face of irritable bowel syndrome. World J. Gastroenterol. 12, 1-5.

Raison, C. L., Dantzer, R., Kelley, K. W., Lawson, M. A., Woolwine, B. J., Vogt, G., Spivey, J. R., Saito,
K., and Miller, A. H. (2009). CSF concentrations of brain tryptophan and kynurenines during immune stimulation with IFN-alpha: relationship to CNS immune responses and depression. Mol. Psychiatry 15, 393-403.

Reinhart, P. H., and Kelly, J. W. (2011). Treating the periphery to ameliorate neurodegenerative diseases. Cell 145, 813-814.

Ruddick, J. P., Evans, A. K., Nutt, D. J., Lightman, S. L., Rook, G. A., and Lowry, C. A. (2006). Tryptophan metabolism in the central nervous system: medical implications. Expert Rev. Mol. Med. 8, 1-27.

Schroecksnadel, K., Winkler, C., Wirleitner, B., Schennach, H., and Fuchs, D. (2005). Aspirin down-regulates tryptophan degradation in stimulated human peripheral blood mononuclear cells in vitro. Clin. Exp. Immunol. 140, 41-45.

Schroecksnadel, S., Sucher, R., Kurz, K., Fuchs, D., and Brandacher, G. (2011). Influence of immunosuppressive agents on tryptophan degradation and neopterin production in human peripheral blood mononuclear cells. Transpl. Immunol. 25, 119-123.

Schwarcz, R., and Pellicciari, R. (2002). Manipulation of brain kynurenines: glial targets, neuronal effects, and clinical opportunities. J. Pharmacol. Exp. Ther. 303, 1-10.

Scully, P., McKernan, D. P., Keohane, J., Groeger, D., Shanahan, F., Dinan, T. G., and Quigley, E. M. (2010). Plasma cytokine profiles in females with irritable bowel syndrome and extra-intestinal comorbidity. Am. J. Gastroenterol. 105, 2235-2243.

Sioud, M. (2006). Innate sensing of self and non-self RNAs by tolllike receptors. Trends. Mol. Med. 12, 167-176.

Spitzer, R. L., Kroenke, K., and Williams, J. B. (1999). Validation and utility of a self-report version of PRIME-MD: the PHQ primary care study. Primary Care Evaluation of Mental Disorders. Patient Health Questionnaire. JAMA 282, $1737-1744$.

Takeuchi, O., and Akira, S. (2010). Pattern recognition receptors and inflammation. Cell 140, 805-820.

Taylor, M. W., and Feng, G. S. (1991). Relationship between interferon-gamma, indoleamine 2,3-dioxygenase, and tryptophan catabolism. FASEB. 5, 2516-2522.

Yu, M., Wang, H., Ding, A., Golenbock, D. T., Latz, E., Czura, C. J. Fenton, M. J., Tracey, K. J., and Yang, 
H. (2006). HMGB1 signals through toll-like receptor (TLR) 4 and TLR2. Shock 26, 174-179.

Zwilling, D., Huang, S. Y., Sathyasaikumar, K. V., Notarangelo, F. M., Guidetti, P., Wu, H. Q., Lee, J., Truong, J., Andrews-Zwilling, Y., Hsieh, E. W., Louie, J. Y., Wu, T., Scearce-Levie, K., Patrick, C., Adame, A., Giorgini, F., Moussaoui, S., Laue, G., Rassoulpour, A., Flik, G., Huang, Y., Muchowski, J.
M., Masliah, E., Schwarcz, R., and Muchowski, P. J. (2011). Kynurenine 3-monooxygenase inhibition in blood ameliorates neurodegeneration. Cell 145, 863-874.

Conflict of Interest Statement: The authors declare that the research was conducted in the absence of any commercial or financial relationships that could be construed as a potential conflict of interest.
Received: 27 March 2012; paper pending published: 11 April 2012; accepted: 26 April 2012; published online: 21 May 2012.

Citation: Clarke G, McKernan DP, Gaszner G, Quigley EM, Cryan JF and Dinan TG (2012) A distinct profile of tryptophan metabolism along the kynurenine pathway downstream of toll-like receptor activation in irritable bowel syndrome. Front. Pharmacol. 3:90. doi: 10.3389/fphar.2012.00090
This article was submitted to Frontiers in Gastrointestinal Pharmacology, a specialty of Frontiers in Pharmacology. Copyright (C) 2012 Clarke, McKernan, Gaszner, Quigley, Cryan and Dinan. This is an open-access article distributed under the terms of the Creative Commons Attribution Non Commercial License, which permits non-commercial use, distribution, and reproduction in other forums, provided the original authors and source are credited. 